\title{
CARTAS ESPAÑOLAS DE JEAN-BAPTISTE SAY: EVIDENCIAS PARA EL ESTUDIO DE LA CIRCULACIÓN DE IDEAS ECONÓMICAS*
}

\author{
JOSÉ M. MENUDO \\ Universidad Pablo de Olavide ${ }^{a}$
}

The Spanish Letters of Jean-Baptiste Say:

Reference Sources in the Diffusion of Economic Ideas

\begin{abstract}
This paper transcribes and annotates five unpublished letters that Manuel María Gutiérrez, Álvaro Flórez Estrada and the Marquis of Valle Santoro respectively sent to Jean-Baptiste Say. An initial hypothesis holds the importance of this correspondence in order to argue the connection of the Spanish authors with the canonical works. We focus on several questions related to the transmission of economic ideas. First, the translation of Say's work is a collective project structured around the Real Sociedad Económica de Amigos del País de Madrid. Secondly, we explain how the translator expurgates the condemnable passages in order to publish Say's Traité déconomie politique in Spanish, those connected to the influence of religion on economic development and public education to be exact.
\end{abstract}

Keywords: Jean-Baptiste Say, History of Economic Thought, Diffusion of economic ideas

JEL Classification: B31, B12, A11

* Received 20 January 2015. Accepted 21 April 2015. Damos las gracias por los constructivos comentarios recibidos, con especial referencia a los tres evaluadores anónimos de esta revista. Cualquier error cometido es responsabilidad nuestra. El autor agradece al Gobierno regional de Andalucía, a través del grupo de investigación Análisis económico aplicado (SEJ-246), y al Centro de estudios andaluces, con el proyecto PRY131/11, la financiación de este trabajo.

a Carr. Utrera km. 1, 41013, Sevilla. E.mail: jmmenpac@upo.es 


\section{RESUMEN}

Este trabajo transcribe cinco cartas inéditas dirigidas a Jean-Baptiste Say por Manuel María Gutiérrez, Álvaro Flórez Estrada y el Marqués de Valle Santoro, respectivamente. Esta correspondencia acredita la proximidad de los autores españoles a las obras canónicas de la Economía clásica. Destacamos algunas evidencias de interés para el estudio de la transmisión de las ideas económicas. En primer lugar, la traducción de la obra de Say es un proyecto colectivo, estructurado en torno a la Real Sociedad Económica de Amigos del País de Madrid. Describimos también la tarea de expurga de los pasajes condenables para poder publicar en castellano el Traité déconomie politique, concretamente aquellos relativos al papel de la religión en el desarrollo económico y en la educación pública.

Palabras clave: Jean-Baptiste Say, Historia del pensamiento económico, Transmisión de ideas económicas

\section{INTRODUCCIÓN}

Cualquier lector interesado en la formación y evolución de las ideas económicas percibe que las contribuciones de los economistas no han tenido la misma importancia en todo tiempo. Sin embargo, puede llegar a pensar erróneamente que en un momento determinado esas ideas sí tuvieron la misma importancia en todo lugar. Precisamente el «enfoque nacional» entre los

historiadores del pensamiento económico indaga, entre otros temas, en las causas del éxito temporal y espacial de las teorías, incorporando las realidades periféricas al estudio de los flujos internacionales de ideas económicas (Lluch 1980; Albertone y Masoero 1994; Cardoso 2003). El enfoque teórico más empleado por esta literatura describe la circulación de ideas como un flujo de información transmitido a la comunidad y que adopta la forma de redes intangibles (Spengler, 1970) ${ }^{1}$. A partir de aquí, las traducciones han sido una de las vías de circulación y divulgación de la literatura económica más estudiadas, especialmente durante la segunda mitad del siglo XVIII y la primera del siglo XIX. Convertidas en un instrumento cuantitativo, han permitido a la literatura tanto elaborar una cronología de la transmisión de

${ }^{1}$ Colander y Coats (1989) elaboran un enfoque general sobre los mecanismos de transmisión de las ideas económicas. Al modelo de Joseph Spengler añaden el modelo de la enfermedad infecciosa, el mercado de las ideas, el enfoque bibliométrico y el enfoque humanístico. A estas propuestas es habitual sumar el modelo de dominación de Albert Hisrchman (1989) y el modelo de los enfoques económico, político y estatal de Peter Hall (1989). Sobre una revisión de la correspondencia entre estos marcos teóricos y los casos nacionales conocidos, vid. Cardoso (2003). 
las ideas como realizar un indicador de la influencia directa de los autores ${ }^{2}$. Menos protagonismo han tenido otros medios de circulación y transmisión de la literatura económica, concretamente los "plagios», las copias manuscritas, los viajeros, los diplomáticos, los salones de lectura o la prensa ${ }^{3}$. Estas vías son, en todo caso, elementos alternativos, paralelos o complementarios a las traducciones y que, lógicamente, también fueron modos de asimilación de ideas foráneas.

Este trabajo emplea uno de los elementos menos atendidos por parte de esta literatura. Se trata de la correspondencia entre los autores de referencia y las realidades periféricas ${ }^{4}$. En concreto, presentamos cinco cartas de autores españoles dirigidas a Jean-Baptiste Say: la carta de Manuel María Gutiérrez de 7 de enero de 1817, la carta de Manuel María Gutiérrez de 23 de mayo de 1817, la carta de Manuel María Gutiérrez de 26 de julio de 1817, la carta Álvaro Flórez Estrada de 19 de septiembre de 1831 y la carta del Marqués de Valle Santoro, sin constancia de la fecha de envío o recepción. No se conocía correspondencia alguna entre los autores españoles y Jean-Baptiste Say aunque el protagonismo del autor francés en el pensamiento económico español señalara en esta dirección. De hecho, la primera mitad del siglo XIX ha sido definida como la era de Say en España (Martín Rodríguez 1989, p. 40), al traer consigo 23 traducciones de las obras de Say y una influencia directa propiciada por el protagonismo que otorga el Plan de estudios de José Antonio Caballero al Traité d'économie politique (en adelante Traité) $)^{5}$.

¿Por qué son importantes estas cartas? En primer lugar, nos permiten situar de forma más clara el lugar de la ciencia económica en España

${ }^{2}$ Carpenter y Redlich (1973) proponen la medición de la influencia por medio de variables temporales como los períodos entre la publicación de los originales y de las traducciones. En el caso de la recepción de Jean-Baptiste Say en España, Menudo y O'kean (2005) aprecian una ausencia de dilación temporal en la aparición de la primera obra de carácter económico de Say en España, a diferencia de otros países - Alemania o Estados Unidos- donde la influencia también fue relevante. El espacio temporal entre la primera y última publicación en castellano es de unos treinta años pero la influencia de Say en España es especialmente intensa durante los veinte años que se sitúan entre 1814 y mediado de los años treinta.

${ }^{3}$ Recientemente, la literatura ha indagado sobre estos mecanismos de transmisión en el caso de España durante los siglos XVIII y XIX: Almenar (1996) ha realizado una aproximación general examinando la difusión de las ideas económicas a través de las publicaciones periódicas españolas de los siglos XVIII y XIX; Astigarraga (2000) presenta la importancia de los plagios, adaptaciones y resúmenes del pensamiento de Necker en España, concretamente en las discusiones sobre el mercado de granos, la Hacienda pública y la organización territorial, mientras Astigarraga y Zabalza (2007) describen la circulación de la obra de Richard Cantillon por medio de plagios; Ramos Gorostiza (2006) analiza a los viajeros británicos — Joseph Townsend y Alexander Jardinepor la atención prestada a las cuestiones económicas, por la capacidad analítica y por la calidad de la información que contienen.

${ }^{4}$ Hay escasos ejemplos de este tipo de trabajos de investigación — v.g. Schwartz y Rodríguez (1983).

5 Sobre la influencia de J.-B. Say en el proceso de institucionalización de la enseñanza de la Economía política en España, vid. López Castellano (2009). 
durante la primera mitad del siglo XIX. Este momento histórico es un cruce de caminos donde se encontrarán corrientes de ideas procedentes de tradiciones propias y ajenas, de novedades analíticas, de innovadoras propuestas de política económica y de revisiones de viejos postulados. En concreto, la proximidad al lugar de privilegio que ocupa Say, uno de los economistas más importantes del siglo XIX y el principal protagonista de la Economía política en el continente europeo ${ }^{6}$, aporta más argumentos para defender la conexión de los autores españoles, sin retraso evidente, con las obras modélicas desde un plano analítico, docente y político. El contenido de la correspondencia contribuye a sostener esta hipótesis - v.g. los textos que el propio Say envía a Manuel María Gutiérrez, el debate planteado a Say por Valle Santoro sobre el modelo del «sistema restrictivo» para el comercio exterior o la consulta sobre la incidencia del gasto público realizada por Say a Álvaro Flórez Estrada ${ }^{7}$. En segundo lugar, esta correspondencia aporta información sobre el pensamiento económico de los autores españoles. Por ejemplo, la asistencia de Álvaro Flórez Estrada a las clases de Economía política de Jean-Baptiste Say en el Athénée de París abre la puerta a la influencia del pensamiento del autor francés en las cuatro ediciones del Curso de Economía publicadas en España $(1835,1840,1848$ y 1852) cuando regresa del exilio ${ }^{8}$. En tercer lugar, encontramos elementos relevantes sobre la historiografía de las traducciones españolas de Say. Hay indicios para sostener que Manuel María Gutiérrez traduce el Catéchisme d'économie politique, con el título Cartilla de Economía política, hasta ahora atribuida a Agustín Pascual ${ }^{9}$. Este y otros elementos presentes en esta correspondencia invitan a presentar la difusión de la obra de Say en España como un proyecto colectivo de la Real Sociedad Económica de Amigos del País de Madrid. Por último, estas cartas señalan algunas vías y obstáculos institucionales a la

${ }^{6}$ Hijo de un comerciante protestante, Jean-Baptiste Say fue un afamado periodista y redactor jefe de La Décade Philosophique, Littéraire et Politique, parlamentario y opositor a Bonaparte, posteriormente empresario y finalmente el primer catedrático de Economía, en el Conservatoire des Arts y a continuación en el Collège de France.

7 Francisco de Paula de Gregorio y Gracia de Tolba nació en Zaragoza el día 12 de octubre de 1786. Luchó joven como teniente de ingenieros en Zaragoza, pasando 5 años en Francia. Vuelve para, tras 4 años en Barbastro, instalarse en Madrid hacia 1817 y llegar a ser vocal de la Real Junta de Fomento de la Riqueza del Reino. Muere en Madrid el 2 de septiembre 1833, antes de la publicación de la última edición de sus Elementos de Economía política aplicados a España.

8 Álvaro Flórez Estrada (1766-1853) nace en Pola de Somiedo (Asturias) y estudia Leyes en Oviedo hasta que se traslada a Madrid donde la incorporación a los círculos políticos le supone un primer y breve destierro. Intentó en 1803 establecer una ferrería en Asturias en una de sus pausas entre los cargos administrativo que ocupó siempre vinculado a los movimientos liberales.

9 Manuel María del Carmen Ignacio Gutiérrez Beganige nace en Cádiz el 16 de julio de 1775 y muere en Madrid en 1850. Reconocido por sus traducciones sobre diversas disciplinas, alcanza la cátedra de Comercio y Economía política de Málaga que involuntariamente sustituye por la Junta Revisora de Aranceles en Madrid. En 1834 abandona la carrera funcionarial para iniciar su vinculación con la Comisión de Fábricas de Hilados, Tejidos y estampados de Algodón del Principado de Cataluña, desde dónde rechaza el librecambio en favor de un modelo denominado «sistema restrictivo». 
difusión de las ideas. Constatan el papel de la información periodística y de los canales diplomáticos en la estructura de difusión y circulación de teorías, ejemplares, borradores e informes. También permiten confirman la tesis de Llombart (2004) a la paradoja que supone una censura creciente acompañada de un incremento de las traducciones; Manuel María Gutiérrez censura para poder publicar y detalla a Say la tarea de expurga de los pasajes condenables.

Este trabajo está estructurado en dos bloques. En primer lugar, analizamos cuestiones relativas al estudio de los flujos internacionales de las ideas económicas presentes en estas cartas. Aunque estos escritos aluden a múltiples aspectos sobre la difusión, hemos delimitado la investigación a las obras de Say en castellano y a los obstáculos a la difusión del pensamiento económico, tratados exclusivamente en las cartas de Gutiérrez, porque son los dos temas que los autores desarrollan en la correspondencia. La segunda parte transcribe las cinco cartas dirigidas a Say, acompañadas de las necesarias anotaciones que permitan una mejor comprensión.

\section{LA PUBLICACIÓN DE LAS OBRAS DE J.-B. SAY EN ESPAÑA}

Manuel María Gutiérrez envía a Jean-Baptiste Say tres cartas de distinta naturaliza e incluso idioma. La primera, enviada el 7 de enero de 1817, es una presentación, la segunda, del 23 de mayo de 1817, es la respuesta a una carta de Say y, finalmente, el 26 de julio de 1817 Gutiérrez envía un tercer escrito que acompaña con la notificación del nombramiento de Say como miembro de la Real Sociedad Económica de Amigos del País de Madrid (RSEM en adelante). Las cuestiones tratadas son diversas pero desarrollaremos los dos asuntos vinculados a la problemática de la circulación de ideas económicas: la traducción de las obras de Say y los obstáculos a la difusión de la ciencia económica.

\subsection{La Traducción de la obra de J.-B- Say}

Manuel María Gutiérrez aprecia una gran diferencia entre las dos primeras ediciones del Traité, 1803 y $1814^{10}$. Comenta en la primera carta que este hecho acontece mientras revisaba las traducciones al castellano de dos obras de Say, Épitomé des principes fondamentaux de l'économie politique

${ }^{10}$ Efectivamente, la diferencia entre la estructura de la primera edición y de las cinco posteriores son tan considerables que Steiner (1998) prefiere hablar de dos obras diferentes. Tras el discurso preliminar, la primera edición se divide en cinco libros: producción (47 capítulos), moneda (17 capítulos), valor ( 9 capítulos), renta (19 capítulos) y consumo (20 capítulos). A partir de la segundo edición, Say introduce la canónica división en tres libros: producción de riqueza (30 capítulos), distribución de riqueza (11 capítulos) y consumo de riqueza (11 capítulos). Ver Say (2006) para una comparación de las ediciones del Traité. 
(1814) y Catéchisme d'économie politique (1815). La segunda era una obra publicada de forma independiente mientras la primera estaba insertada, por primera vez y de forma constante en las sucesivas ediciones, como punto final a la segunda edición del Traité (1814). El resultado de la evaluación es una desaprobación de ambos textos por su mala calidad. El primer documento era una traducción del Épitomé, realizada por Manuel Antonio Rodríguez $^{11}$, que finalmente ambos publicarían en $1817^{12}$. La referencia al Catéchisme plantea más dudas porque en 1816 aparecieron dos traducciones al castellano, los Principios de Economía Política y la Cartilla de Economía Política. La primera obra únicamente añade una dedicatoria a los directores de la Real Compañía de Filipinas firmada por Cesáreo María Sáenz de la Barrera, mientras la Cartilla, rubricada por Agustín Pascual, incorpora un prólogo del traductor. Esta última obra será vendida, junto al Epitome, como parte del cuarto volumen que acompaña a la traducción del Traité ${ }^{13}$. Este hecho nos permite platear que Gutiérrez se refería a la Cartilla porque a este conjunto de volúmenes hace referencia en la carta a Say de 23 de mayo de 1817:

A este fin he puesto en manos de los Señores Aguirre[,] del comercio de esta plaza, un ejemplar completo de mi traduccion en 4 volumenes en pasta. Los 3 primeros comprenden su traducción, y el $4^{\circ}$ el epitome y la cartilla, pues no he tenido noticia de la dirección que se ha dado al otro ejemplar que remití á Bayona ${ }^{14}$.

La revisión de ambas traducciones debió ser tal que dio lugar a una reelaboración de ambos textos. Por esta razón, Gutiérrez afirma en esta correspondencia que el autor de la Cartilla de Economía Política es él.

11 Poco conocemos de esta figura. En las ediciones de Say, Manuel Antonio Rodríguez aparece como miembro de la Academia General de Ciencias, Bellas Letras y Nobles Artes de Córdoba y de la Sociedad Económicas de Amigos del País de Córdoba. En la Academia General presentó su obra $L a$ Musa patriótica ó laureles del General Wellington y de las naciones aliadas y la traducción de Chiare, Fresche é Dolci Acque \&. Ocupó un puesto en el Departamento de Fomento general del Reino y Balanza del comercio y, posteriormente, en la Secretaría de Estado y del Despacho de Hacienda y Secretario del Rey, desde donde fue propuesto para formar parte de la Comisión de aranceles en 1824. También fue miembro de la diputación permanente de la RSEM.

12 El Epítome de los principios fundamentales de la economía política (1817) fue publicado como obra independiente, tanto en la Imprenta de Collado de Madrid como en la Imprenta de la Real Compañía (Castro Valdivia, 2013).

134 tomos en $8^{\circ}$. Tomos 1, 2, 3: Tratado de economía política, o simple exposición del modo con que se forman, distribuyen y consumen las riquezas. Refundido por el mismo y aumentado con un epitome que comprende los principios fundamentales de la economía política y una tabla analítica de materias. Trad. M. M. Gutiérrez y M.A. Rodríguez. Madrid, Imprenta de Collado, 1816. Tomo 4: Epitome de los principios fundamentales de la economía política. Trad. M. A. Rodríguez y M. M. Gutiérrez. Madrid, Imp. de Collado, 1816. (junto con:) Cartilla de economía política, o instrucción familiar, que manifiesta como se producen, distribuyen y consumen las riquezas. Obra fundada en hechos y útil a toda clase de personas. Trad. Del francés por A. Pascual. Madrid, Imp. de la Real Compañía, 1816.

14 Infra. 
Señalaros, Señor, que el Cathéchisme [sic] ha sido publicado con un nombre distinto al mío, y que la obra principal lo ha sido con el nombre de Rodríguez y con el mío. El Sr. Rodríguez solo había comenzado esta traducción pero, por una condescendencia necesaria en las circunstancias actuales, no me ha sido permitido mortificar el amor propio de estos dos individuos ${ }^{15}$.

Podemos señalar algunas evidencias que sustentan la afirmación de Gutiérrez. En primer lugar, el perfil profesional de Agustín Pascual catedrático de Zoofisiología de la real escuela veterinaria, reconocido autor en el ámbito de la Botánica y sin constancia alguna de obras relacionadas con la Economía más allá de algunas reseñas sobre cartillas agrarias- no se ajusta al contenido de la Cartilla $^{16}$. El prólogo del traductor evidencia importantes conocimientos de la ciencia económica, con la mención de Adam Smith y de Antoine-Louis-Claude Destutt de Tracy, autor traducido precisamente por Gutiérrez en $1817^{17}$. En segundo lugar, se trata de una traducción de mejor calidad que las precedentes, al igual que ocurre con el Traité, y con la misma solución a los problema que conlleva la formalización de una lengua de especialidad — por ejemplo, la elección del término empresario en lugar de fabricante, maestro u oficial o la opción del adjetivo mercantil en lugar de comercial ${ }^{18}$. Añadimos que ambos textos son definidos como parte del proyecto docente que Say propone - en concreto, el Catéchisme era un texto para los jóvenes ${ }^{19}$. La RSEM puede ayudar a explicar por qué Gutiérrez no figura como autor de la Cartilla. Agustín Pascual fue admitido como miembro el 26 de enero de 1811 y tuvo un papel destacado en distintas áreas ${ }^{20}$. Gutiérrez se incorpora a la institución el 14 de diciembre de 1814 y como consecuencia de su traducción de Say: «Para recompensar los servicios que he dado a mi Nación, traduciendo su obra, la Sociedad

\section{Infra.}

16 En 1817, la RSEM convocó un premio sobre cartillas agrarias y Agustín Pascual García (1786-1822) elabora un informe sobre una cartilla rural, destacando su vinculación con la línea de pensamiento iniciada por Jovellanos sobre esta materia (Montagut 2004, pp. 87-101). Colaborador en el periódico Semanario de Agricultura, ayudó a editar Agricultura general (1819) de Alonso de Herrera. Sobre la obra de Agustín Pascual, ver Misas (1996).

17 Principios de Economía Política: considerados por las relaciones que tienen con la voluntad humana (2 volúmenes), Madrid, Imprenta del Cano, 1817.

18 Sobre el análisis de las traducciones españolas de Jean-Baptiste Say, ver Menudo y O'kean (2005) y Hoyos (2015).

19 La expresión empelada en la Cartilla de Economía Política será «como para la enseñanza de los jóvenes», mientras "poniéndolo al alcance de la juventud» es la traducción de la carta de 7 de enero de 1817.

20 Archivo de RSEM, legajo 214, documento 5/1. Entre 1815 y 1820 fue censor de la RSEM. Su participación en el concurso de la cátedra de Economía política y Estadística de la institución en 1820, junto al primer catedrático José Felipe de Olive, y la elección de la Cartilla de Economía Política como manual señalan que Agustín Pascual era una figura importante en las cuestiones institucionales relacionadas con la ciencia económica (Moral y Colmenero 2006, p. 97). 
de Económica política de Madrid me acaba de admitir en su seno» ${ }^{21}$. Ambos debieron establecer estrechos vínculos porque, junto a su papel como economista, Gutiérrez pasa a convertirse en uno experto en ganadería en España $^{22}$.

La correspondencia entre Say y Gutiérrez también discute sobre la traducción de las ediciones del Traité. En la carta de 23 de mayo de 1817, Gutiérrez hace referencia a la tercera edición del Traité, única de la que no se publicó ninguna traducción ${ }^{23}$. Presenta la futura reedición de su traducción como una adaptación a la tercera edición, y no como la reimpresión que la literatura ha presentado hasta ahora. «Me ha sido igualmente sensible que á $\mathrm{V}$., el no haber hecho la traducion de su hermoso tratado de Economia por su $3^{\text {a }}$ edición, aunque la estoy rectificando con presencia de ella, y espero publicarla mas adelante con las notables mejoras que tiene ${ }^{24}$. En la carta de julio, Gutiérrez hace referencia al trabajo de revisión de su traducción en presencia de la tercera edición del Traité: «Continúo limando, corrigiendo, y traduciendo el mismo tratado, con presencia de su $3^{\mathrm{a}}$ edicion, é inmediatamente que se concluya y publique, enviaré á V. por medio del mismo Sr. Consul General de éstas tres provincias, un egemplar completo ${ }^{25}$. Sin embargo, una comparación de las dos ediciones, de 1816 y de 1817, demuestra que la tarea señalada por Gutiérrez no llegó a materializarse y, en consecuencia, se llevó a cabo una reimpresión de la segunda edición en lugar de la traducción de la tercera edición.

En suma, la correspondencia de Gutiérrez con Jean-Baptiste Say señala a la RSEM como una institución central para entender la difusión directa de la obra de Say en España. Los traductores del Traité eran, salvo Juan Sánchez Rivera, miembros de esta sociedad y ahora conocemos que los borradores eran revisados entre ellos ${ }^{26}$. A la cuestión de la firma de los textos y a la circulación de borradores entre los miembros de la RSEM, hemos de añadir

21 Infra.

22 Manuel María Gutiérrez tradujo la obra de Guillaume Louis Ternaux, Memorias sobre la utilidad de la importación y cría en Francia del ganado lanar (1831) y el volumen de Charles Dupin, Lecciones para el propietario territorial ó el labrador del Barón Carlos Dupin (1832). En 1835 publicó su Informe sobre el ganado merino: castración, exportación y otras cuestiones dado al Estamento de Ilustres Próceres y, entre sus artículos en Cartas españolas, encontramos «Algodón en rama, Arroz secano de Filipinas y de Puerto-Rico, ó arroz de monte» (Tomo 5, 1832). Sobre esta bibliografía de Manuel María Gutiérrez, ver Antón Ramírez (1865).

23 De la cuarta edición aparece una traducción de Juan Sánchez Rivera con el mismo prólogo de Manuel $\mathrm{M}^{\mathrm{a}}$ Gutiérrez que se publica en 1821 (en Madrid y en Burdeos) con la inclusión de las Cartas de Say a Mathus. De la quinta y última edición en vida del autor existe una traducción de José Antonio Ponzoa, con el acompañamiento de La vida de Say por C. Compte, publicada en 1838.

24 Infra.

25 Infra.

26 José Queipo de Llano es miembro de la RSEM desde su fundación en 1775 (Sancha 1780, p. 315) y José Antonio Ponzoa, el último traductor del Traité, será el titular de la Cátedra de Economía política de la Sociedad Económica Matritense desde 1820. 
la defensa que Gutiérrez realiza frente a Say de la desafortunada traducción del Traité a cargo de José Queipo de Llano ${ }^{27}$.

En cuanto al juicio que hace el Sr, Bachiller del merito de la traduccion, aunque me parece que por la fecha en que se escribió, habla del Sr. Queypo, lo miro como absolutamente falso; porque es una de las traducciones, en mi concepto, que le han hecho en este pais con mas atencion y detenimiento. Así, me créo obligado en su ausencia á defenderle cuando se me presente la ocasión, y á lavar tambien á mi maestro de lo que se le quiere hacer decir y nunca ha dicho ${ }^{28}$.

Este conjunto de hechos señala que la traducción de la obra económica de Say es un proyecto colectivo de la RSEM donde Gutiérrez toma el papel de interlocutor. Por un lado, Gutiérrez demanda con insistencia a Say textos, publicados o manuscritos, para que puedan ser traducidos al castellano y como respuesta Say le envía De l'Angleterre et des Anglais (1815) el 7 de abril de $1817^{29}$. Por otro lado, Gutiérrez presenta a la RSEM como el elemento dinamizador que permita impulsar la docencia de Economía política y, con ello, derribar las instituciones que impiden la prosperidad de España.

Feliz por haber obtenido del gobierno el reconocimiento de la necesidad de establecer en este reino las cátedras de Economía política y de Comercio, la Academia de Madrid se dispone a hacer todos los esfuerzos para que esta ciencia sea enseñada, siguiendo sus excelentes principios, y para que su útil propagación disipe, poco a poco, los funestos errores y los prejuicios enraizados que, para nuestro perjuicio, retrasan desde hace mucho tiempo la prosperidad de este país ${ }^{30}$.

\subsection{La censura y los obstáculos a la traducción}

La correspondencia de Manuel María Gutiérrez aborda la cuestión de las instituciones como obstáculos a la circulación de ideas. El protagonismo de la censura gubernativa y el control inquisitorial de las publicaciones en la

27 En una comparación de las cuatro traducción del Traité publicadas en el siglo XIX, el resultado de José Queipo de Llano (1804-07) es claramente de menor calidad que las ediciones posteriores (Menudo y O'kean 2005, p. 123).

28 Infra.

29 De la Inglaterra y de los ingleses. Por Juan Bautista Say, autor del Tratado de Economía Política. Traducido del francés al castellano por D. P. M. O., Madrid, Imprenta de Collado, 1817. Es posible que el traductor fuese Pedro María Oliver, editorialista de Efemérides de la Ilustración de España, de Minerva y traductor también de la obra de Alexander von Humboldt, Minerva: ensayo político sobre el reino de Nueva España, sacado del que publicó en francés Alexandro de Humboldt por D.P.M. de O., Madrid, Imprenta de Núñez, 1818, 2 vol.

30 Infra. 
decadencia de España fue creada principalmente por ilustrados y viajeros franceses y británicos (Iglesias 1998; Bolufer 2003). Diversos estudios han señalado como la incapacidad de los controles, la escasa formación de los censores o la indolencia hicieron permeables las barreras y permitieron que las traducciones se convirtieran en importantes vías de penetración del pensamiento europeo (Defourneaux 1973; López 1995; Domergue 2003). A pesar de ello, las traducciones de Economía se vieron afectadas y limitadas por las instituciones censoras, como ocurría también con el resto de las publicaciones y en el resto de Europa. La cuestión es cómo esas barreras han influido en la circulación de textos económicos y en la penetración de ideas ilustradas.

El fenómeno del incremento de las traducciones, desde la segunda mitad del siglo XVIII y como consecuencia del aumento general de la literatura ensayística, solo podría explicarse porque los textos elegidos no fueran peligrosos para la doctrina oficial o bien porque la propia traducción expurgara los pasajes condenables. Llombart (2004) sostiene que la incidencia de los obstáculos fue mínima al optar por autocensurar para poder traducir. La traducción de Economía política amplía el número de receptores del texto inicial, abarata el precio del libro en el país de destino, por lo que puede tener más lectores que el original, y difunde la obra del autor. Una creciente demanda empujó a los traductores a abordar la tarea de convencer a los censores, principalmente expurgando determinados pasajes críticos para conseguir que fuera publicable en España en unas condiciones de falta de libertad de expresión. Una tesis alternativa explica el incremento de las traducciones como consecuencia de una política del régimen absolutista (Reeder 1978). Se trata de la función censora de las traducciones de Economía que convierte los textos discrecionalmente manipulados en un instrumento al servicio del poder.

En su correspondencia con Say, Gutiérrez reconoce la necesidad de censurar para poder publicar. En primer lugar, la finalidad principal de las traducciones españolas no es otra que la recepción de los conocimientos económicos que consideraba necesario difundir entre los lectores españoles.

He estado tentado de rehacer estas traducciones, a pesar de mis numerosas ocupaciones. He creído tener que hacerlo, porque la lectura de su nuevo tratado me ha convencido de que este había sufrido grandes cambios con relación a la publicación que usted había hecho en el año 1803 y porque, de todas las naciones de Europa, la nuestra es la que sufre la más urgente necesidad de conocer los principios de una ciencia tan generalmente útil ${ }^{31}$.

Defensor del fenómeno europeo de la circulación internacional de ideas económicas que permite la multiplicación de la literatura y, en consecuencia,

${ }^{31}$ Infra. 
la mejora tanto en el análisis económico como en la capacidad reformadora de la política económica de los gobiernos, Gutiérrez reivindica la necesidad de incorporar la realidad de la cultura receptora al proceso de difusión geográfica.

Por lo tanto, para no privar a mis conciudadanos de la lectura tan útil de su interesante obra tengo la determinación de suprimir algunos pasajes, a mi pesar, que no serían parte de los elementos de esta ciencia y que habrían probablemente impedido la publicación de mi traducción; en otros voy simplemente a suavizar algunas expresiones, que habrían sido demasiado fuertes para este país, o a ocultar algunas ideas cuya audacia habría satirizado demasiado claramente para lo que nosotros generalmente admitimos ${ }^{32}$.

En segundo lugar, Gutiérrez adopta el papel de autor de la obra en el país de destino y practica lo único que puede hacer para publicar sin libertad de expresión. El traductor autocensura la obra escrita, originalmente en las condiciones políticas de Francia, para hacer viable su publicación en un marco institucional como el español. Adapta el texto, con las modificaciones que ello comporta, mientras en otras ocasiones elimina partes que considera poco importantes para el riesgo que conllevan. En su correspondencia detalla los dos temas que le obligan a modificar el texto original: el desarrollo económico (capítulo XI del libro II) y los gastos públicos, concretamente la enseñanza pública (capítulo VI del libro III).

Por ejemplo, lo que usted dice sobre los bienes territoriales de las manos muertas, y sobre los inconvenientes de enseñar los principios de la moral por medio de los libros. Sobre todo lo demás, me esfuerzo por reflejar en nuestra lengua sus ideas y sus expresiones, con la claridad y la elocuencia que reinan en vuestra obra; pero debo vanagloriarme poco de tener este placer ${ }^{33}$.

Say había empleado las páginas dedicadas a la población para exponer su teoría del progreso económico, discutiendo particularmente la situación de España, las causas de su retraso económico y las medidas para su industrialización. Será en los cursos de Economía impartidos en el Athénée de París donde Say dedique la mayor parte de la sesión sobre población a explicar la decadencia de España. En primer lugar, subraya el papel de la Inquisición en la «destrucción» de las fuentes que procuran los recursos y, en consecuencia, de la producción. La expulsión de moriscos y judíos provocó una reducción del capital humano, del capital físico que les acompañaba

\footnotetext{
32 Infra.

${ }^{33}$ Infra.
} 
en su marcha y de las redes de financiación, mientras los tribunales inquisitoriales generaban una inseguridad jurídica generalizada. En otros textos añade dos efectos negativos de la religión católica sobre las capacidades productivas. Gutiérrez no tiene que lidiar con el primero porque aparece en la tercera y cuarta edición de Traite ${ }^{34}$. Las decisiones productivas deben estar basadas en competencias adquiridas por la educación industrial y por la familia. La desaparición del juicio por causa de la religión o de la superstición limita la producción y aletarga las capacidades. El segundo argumentos aparece en la segundo edición y será alterado por Gutiérrez. El celibato religioso y la consiguiente acumulación de patrimonio generan una ociosidad de los recursos en manos de la Iglesia que limita la capacidad productiva. La comparación con el texto original de Say evidencia el grado de modificación realizado por Gutiérrez.

Se han quejado mucho del perjuicio que los conventos hacen a la población, y con razón; pero se han equivocado sobre las causas porque no es el celibato religioso quien hace este mal, es su ociosidad. Se dice que ellos hacen trabajar sus tierras: ¡bonita cosa! ¿Las tierras se quedarían incultas si los monjes llegasen a desaparecer? Al contrario, donde quiera que los monjes han sido reemplazados por talleres de industria, de lo que hemos visto muchos ejemplos en la revolución francesa, el país ha ganado, tanto en productos de su agricultura como de su industria manufacturera; y siendo de este modo mayor el valor total producido, la población de estos países ha aumentado ${ }^{35}$.

Las manos que no trabajan por sí las tierras perjudican, también infinito á la población, porque en ellas no producen lo que pudieran. Se me dirá acaso: pero estas manos cultivan, ó hacen cultivar sus tierras. ¡Excelente argumento! ¿Y aunque faltasen, quedarían por esto baldías? Todo lo contrario. Donde quiera que se han reemplazado por fábricas y talleres de industria, el país ha ganado, además de los mismos productos rurales, los de su industria fabril ${ }^{36}$.

Para Say, la eliminación de privilegios y reglamentaciones de las instituciones arcaicas es una condición necesaria pero no suficiente porque el crecimiento económico ampliaría la desigualdad hasta acabar con la

34 «La religión y las costumbres influyen también en la población, únicamente a causa de su influjo en la producción. Por eso siendo las costumbres de los países protestantes más favorables a la producción, estos países no sólo están más abastecidos que los países católicos, sino que son más populosos. Es lo que notan todos los que viajan» (Say 1821, II, p. 147). Aunque este párrafo solo aparezca en la tercera y cuarta edición del Traité, el Cours complet d'économie politique pratique incorpora y desarrolla la cuestión, aclarando que se trata de Irlanda y España (Say 1840, p. 141).

${ }^{35}$ Say (1814, II, p. 154, traducción propia).

36 Say $(1816$, II, p. 416). 
armonía social que la prosperidad requiere. En el pensamiento sensualista, un sistema generalizado de instrucción pública es indispensable para que la población disfrute plenamente de los derechos naturales y reafirme su independencia respecto a las élites. Frente al concepto de educación ilustrada, la instrucción pública reduce las diferencias sociales en capital humano, en riqueza y en intereses. Say considera que, a pesar de una aparente diversidad y oposición, el verdadero interés de los individuos es fundamentalmente armonioso y la formación será el medio de reconocerlo. Por lo tanto, la instrucción industrial sirve a la nación como medio para lograr la estabilidad social y como recurso para generar crecimiento económico. El objetivo es una organización de la sociedad donde la prosperidad y el bienestar de la nación reposan en la actividad de los gobernados, y no en los gobernantes. Esto explica que Say empiece a construir su edificio teórico por la Economía política, y no por la Moral o la Política ${ }^{37}$. Gutiérrez no se atreve a ir tan lejos, desligando la moral de toda autoridad.

El único estudio importante que no me parecería objeto de enseñanza pública es el estudio de la moral. ¿Es necesario que un profesor nos diga lo que le debemos a nuestro padre, a nuestros hermanos y hermanas, a nuestros amigos? La moral debe aprenderse en cualquier sitio y no se enseña en ningún lugar ${ }^{38}$.

Lo que exige menos enseñanza, son aquellos principios de moral universal, que parece que están grabados en todos los corazones. ¿Qué necesidad hay, por ejemplo, de inculcar en el espíritu de la juventud el respeto y veneración que debemos tener á nuestros padres, hermanos y amigos? Esta parte de la moral está dentro de nosotros mismos: la aprendimos de todos los seres que nos rodean, y basta para aprenderla el ejercicio de la razón: no necesita de maestros, porque es muy dulce su práctica cuando nuestro corazón no está viciado ${ }^{39}$.

Cinco años más tarde, en 1821, las alteraciones por autocensura han desaparecido y la traducción del Traité de Juan Sánchez Rivera, reeditada en 1836 y en 1839, ya es fiel al original. Lo mismo ocurre con la traducción de José Antonio Ponzoa, publicada en 1838.

En resumen, la censura no era ni una función ni una finalidad de las traducciones. Simplemente se trataba de una exigencia impuesta por la falta de libertad. El Tratado de Economía Política de Say se suma a aquellas obras - por ejemplo, la Ciencia de la Legislación de Gaetano Filangieri o la Riqueza de las

\footnotetext{
37 Vid. Steiner (1990).

38 Say (1814, II, pp. 273-4, traducción propia).

39 Say (1816, III, pp. 141-2).
} 
naciones de Adam Smtih - en las que sus traductores - Jaime Rubio en 1787-1789 y José Alonso Ortiz en 1794- expurgan determinados pasajes críticos para conseguir que la traducción fuera publicable en España (Astigarraga 2004; Lasarte 1976). En el caso de Manuel María Gutiérrez, la demanda de un entorno ávido de publicaciones es más evidente. Así se desprende de esta correspondencia, donde aparecen frecuentes peticiones de textos, publicados o no en Francia, que puedan ser traducidos y publicados en España, a pesar del riesgo que esta empresa siempre conllevaba.

A pesar de todas las precauciones que demanda la prudencia he entregado esta traducción a la imprenta con algún peligro porque es difícil separar los intereses personales del resentimiento cuando las verdades ofenden y porque aquellos que viven en el error toman la dulce costumbre de amarlo y cuidarlo.

\section{CINCO CARTAS DIRIGIDAS A J.-B. SAY}

Presentamos cinco cartas halladas en el legajo NAF 26252 del Fondo Jean-Baptiste Say de la Biblioteca nacional de Francia. Todas ellas están transcritas en castellano, para lo que ocasionalmente ha sido necesario realizar la traducción del francés. Es el caso de la carta de Manuel María Gutiérrez de 7 de enero de 1817 y de la carta del Marqués de Valle Santoro. El orden de presentación es cronológico aunque incorporamos en último lugar la carta sin fecha del Marqués de Valle Santoro.

La transcripción es ne varietur. El principio de transcripción es diacrónico, y no diplomático; nos hemos esforzado por reconstruir la cronología de la redacción de las cartas más que por seguir su apariencia gráfica. Las intervenciones han sido enmarcadas por corchetes ( [ [ ] ») y las transcripciones inciertas están seguidas de « [?] ».

\subsection{Carta primera de Manuel María Gutiérrez}

Santander 7 de enero de 1817

Don Manuel María Gutierrez

Señor Jean Baptiste Say.

Ignoraba que usted hubiera publicado su nuevo tratado de economía política $^{40}$, cuando fui encomendado a examinar las traducciones al español de su resumen de esta obra y de vuestro Cathéchisme $[\mathrm{sic}]^{41}$; traducciones que no me ha sido posible aprobar porque me han parecido poco dignas del autor original.

40 La segunda edición del Traité d'économie politique (1814).

41 Se trata del Épitomé des principes fondamentaux de l'économie politique, publicada junto a la segunda edición del Traité d'économie politique (1814), y del Catéchisme d'économie politique (1815). 
He estado tentado de rehacer estas traducciones, a pesar de mis numerosas ocupaciones. He creído tener que hacerlo, porque la lectura de su nuevo tratado me ha convencido de que este había sufrido grandes cambios con relación a la publicación que usted había hecho en el año 1803 y porque, de todas las naciones de Europa, la nuestra es la que sufre la más urgente necesidad de conocer los principios de una ciencia tan generalmente útil ${ }^{42}$. No le repetiré más Señor, lo que toda Europa ha visto antes que yo; que la economía política es, desde que Smith le ha proporcionado un alfabeto, verdaderamente una ciencia reducida a principios que se ha convertido bajo su pluma en una lengua elocuente y perfeccionada. He creído, por lo tanto, prestar un servicio importante a mi patria mostrándole el precioso tratado que todas las Naciones civilizadas de Europa le adeudan en tanto que sus principios solo son consecuencia necesarias del orden invariable de la naturaleza, y lo son igualmente de todas las edades y de todos los países.

Por lo tanto, para no privar a mis conciudadanos de la lectura tan útil de su interesante obra tengo la determinación de suprimir algunos pasajes, a mi pesar, que no serían parte de los elementos de esta ciencia y que habrían probablemente impedido la publicación de mi traducción; en otros voy simplemente a suavizar algunas expresiones, que habrían sido demasiado fuertes para este país, o a ocultar algunas ideas cuya audacia habría satirizado demasiado claramente para lo que nosotros generalmente admitimos. Por ejemplo, lo que usted dice sobre los bienes territoriales de las manos muer$\operatorname{tas}^{43}$, y sobre los inconvenientes de enseñar los principios de la moral por medio de los libros ${ }^{44}$. Sobre todo lo demás, me esfuerzo por reflejar en nuestra lengua sus ideas y sus expresiones, con la claridad y la elocuencia que reinan en vuestra obra; pero debo vanagloriarme poco de tener este placer.

A pesar de todas las precauciones que demanda la prudencia he entregado esta traducción a la imprenta con algún peligro porque es difícil separar los intereses personales del resentimiento cuando las verdades ofenden y porque aquellos que viven en el error toman la dulce costumbre de amarlo y cuidarlo. Le he enviado, Señor, el primer ejemplar; os lo doy como si fuese mi maestro y como testimonio de mi estima y de mi reconocimiento: lo haré llegar a Bayona en el primer navío que parta desde aquí hacia ese destino; le llegará a título gratuito.

Toda mi ambición es que obtenga su aprobación. En todo caso, recibiré sus observaciones como instrucciones precisas de las que me apresuraré a sacar provecho para la utilidad general.

42 Tratado de Economía Política o exposición simple del modo cómo se forman, distribuyen y consumen las riquezas. Por Juan Bautista Say. Refundido por el mismo y aumentado con un epitome que comprende los principios fundamentales de la economía politica y una tabla analítica de materias. Traducido al castellano por Don Manuel María Gutiérrez y Don Manuel Antonio Rodríguez, Madrid, Imprenta de Collado, 3 tomos, 1816.

43 Say 1814 , II, p. 154.

44 Say 1814, II, pp. 273-4. 
Señalaros, Señor, que el Cathéchisme [sic] ha sido publicado con un nombre distinto al mío, y que la obra principal lo ha sido con el nombre de Rodríguez y con el mío. El Sr. Rodríguez solo había comenzado esta traducción pero, por una condescendencia necesaria en las circunstancias actuales, no me ha sido permitido mortificar el amor propio de estos dos individuos.

Leo en el Journal des débats ${ }^{45}$, del 21 del mes pasado, que acabáis de iniciar un curso de economía política ${ }^{46}$. Como estoy convencido de la gran importancia que debe tener una obra salida de vuestras manos sobre esta ciencia, de la que usted es el creador, oso suplicaros con insistencia entregar al portador de esta carta un ejemplar de su discurso de apertura, si está impreso, o una copia de vuestro manuscrito si no lo está ${ }^{47}$. El precio del ejemplar, o lo gastos del amanuense[,] os serán pagados inmediatamente. Oso contar con ese favor, Señor; los hombres de un mérito real y extraordinario están obligados a ampliar sus conocimientos [en] todas las Naciones.

Para recompensar los servicios que he dado a mi Nación, traduciendo su obra, la Sociedad de Económica política de Madrid me acaba de admitir en su seno ${ }^{48}$; me ha encargado también la redacción de tres memorias: las dos primeras están acabadas y la tercera solo está esbozada.

La primera de estas memorias trata sobre los inconvenientes y los errores ligados a las Corporaciones de oficios y maestrías; el segundo ofrece un método razonado de enseñanza de la Ciencia del comercio, y de llevar los libros de contabilidad doble; la tercera, por último, trata igualmente de un método razonado de enseñar economía política según los principios de su Cathéchisme [sic] poniéndolo al alcance de la juventud ${ }^{49}$.

Feliz por haber obtenido del gobierno el reconocimiento de la necesidad de establecer en este reino las cátedras de Economía política y de

45 Journal des débats politiques et littéraires (1814-1944). El anuncio aparece el 9 de noviembre de 1816: «L'Athénée royal vient de publier le programme de ses Cours pour l'année 1817: On y remarque, parmi ses professeurs: M.M. Thénard, pour la Chimie; Say, pour l'Economie politique; Trémery, pour la Physique; Buttura, pour la Littérature italienne; Hippolyte Cloquet, pour la Physiologie; Pariset, pour l'Entendement humain; Rougier de la Bergerie, pour l'Agriculture et la Physique végétale; Brès, pour la Physique appliquée aux beaux-arts; Michel Berr, pour la Littérature allemande, etc. etc.».

46 El contenido del curso, impartido durante tres años académicos (1815-1816, 1816-1817 y 1818-1819), ha sido editado por Philippe Steiner, Cours d'économie politique et autres essais (París: Flammarion, 1996).

47 Los discursos de apertura de los cursos impartidos por Say no fueron publicados en vida del autor. En la primera edición de sus obras de 1848 aparecen publicados los discursos de apertura impartidos en el Conservatoire des Arts et Métiers $(1820,1828)$ y en el Collège de France (1830 y 1831).

48 Gutiérrez fue admitido como miembro de la Real Sociedad Económica Matritense de Amigos del País el 14 de diciembre de 1814.

49 Se trata del Plan de enseñanza de Comercio, Partida simple y doble y Economía política que Gutiérrez había elaborado para el Consulado de Alicante y entregado en diciembre del año 1814 (Archivo de la RSEM, Legajo 235. Documento 28). Como el consulado había iniciado la enseñanza antes de recibir el plan, la RSEM considera conveniente adoptarlo como referente futuro (actas de la RSEM de 21 de diciembre de 1816). 
Comercio $^{50}$, la Academia de Madrid se dispone a hacer todos los esfuerzos para que esta ciencia sea enseñada, siguiendo sus excelentes principios, y para que su útil propagación disipe, poco a poco, los funestos errores y los prejuicios enraizados que, para nuestro perjuicio, retrasan desde hace mucho tiempo la prosperidad de este país.

Sabe usted, Señor, que sus conocimientos pueden ser extremadamente útiles para una nación que le reclama, por mi voz, la participación. $\mathrm{Si}$, en consecuencia, usted aprueba que yo sea su fiel intérprete, usted se dignará a tomar la molestia de transmitirme aquellas ideas suyas que juzgue más útiles para mi tercera memoria, que me presenta algunas dificultades y por la que no oso confiar en mis posibles conocimientos; tendrá igualmente la bondad de indicarme las obras que todos los profesores deberán estudiar para estar a la altura de sus importantes obligaciones y para combatir con firmeza los frecuentes errores incluidos en todos los tratados de economía política.

Ignoro finalmente Señor, en este obscuro retiro donde me ha relegado el deseo de disfrutar de una sombra de la libertad, si ha publicado alguna otra obra antes o después de la publicación de su tratado. En ese caso, osaría rogaros enviarme también un ejemplar a la persona que me hace el favor de presentaros esta carta y que será encargada de pagaros el importe.

Oso vanagloriarme, Señor, de la esperanza de que usted me haga el honor de responderme, dirigiéndome su carta con la cobertura de M. Grislain, Consul de Francia en Santander ${ }^{51}$.

Con mi consideración distinguida me cabe el honor de suscribirme de Ud., muy Señor mío,

Su muy humilde y seguro servidor,

Manuel María Gutierrez

\subsection{Carta segunda de Manuel María Gutiérrez}

Santander 23 de Mayo de 1817

Don Manuel Gutierrez

Señor Dn. Jean Baptiste Say

Muy Señor mio, acabo de recibir con mucho agrado la apreciable carta de V. de 7 de Abril, detenida en Burdeos por la persona á quien la entregó el Señor Daniel.

50 Real decreto de 9 de junio de 1815: «Real decreto establece SM en todos sus reinos las Sociedades económicas, y describe las reglas con que en lo sucesivo han de gobernarse para su uniformidad y reunión.» Fermín Martín de Balmaseda, Decretos del Rey Don Fernando VII, tomo II. Madrid: Imprenta Real, 1819, pp. 410-413.

51 Louis-François Graslin (Nantes, 1769-Bourg-la-Reine, 1850) era hijo del conocido crítico fisiócrata Jean-Joseph-Louis Graslin. Fue cónsul de Francia en Santander durante veintiún años, desde 1816 hasta 1837, y autor de De l'Ibérie, ou Essai critique sur l'origine des premières populations de l'Espagne (1838). Sobre Louis-François Graslin, ver Bladé (1870). 
Me ha sido igualmente sensible que á V., el no haber hecho la traducion de su hermoso tratado de Economia por su $3^{\mathrm{a}}$ edición ${ }^{52}$, aunque la estoy rectificando con presencia de ella, y espero publicarla mas adelante con las notables mejoras que tiene ${ }^{53}$.

No me atreveré á asegurar que mi traduccion sea la espresión fiel de las ideas de V; porque conozco prácticamente las dificultades que ofrece esta especie de trabajo; pero me parece que no las he alterado esencialmente, lo que basta paraque pueda ser entendidas de todos, habiendo procurado esplicarme con sensillez y claridad. Ha sido mi modelo otra traduccion de D. José Queypo á quien conozco personalmente, y de cuyos conocimientos en la ciencia, y en las dos lenguas, no se puede dudar ${ }^{54}$.

Sin embargo, he visto en un Mercurio de esa Capital del mes de Febrero de este año, que un Bachiller de Salamanca mienta á V. y á su traductor, asegurando que V. ha dicho en su Carta-dedicatoria á el Emperador Alejandro, "que á no haber entrado en Paris con sus numerosos egercitos, ignoraria la Europa el verdadero estado de la ciencia,» lo cual no han visto ciertamente mis ojos, y añadiendo, «que sus obras aunque mal traducidas en Castellano, éran seguidas con gran veneracion en este pais» ${ }^{55}$.

Esta última circunstancia es demasiado cierta, dígase ó nó irónicamente; porque el gobierno protege cada días más las catedras de Economia politica, donde se enseña la ciencia por su tratado, y yá se advierte que sus

52 Traité d'économie politique, ou Simple exposition de la manière dont se forment, se distribuent et se consomment les richesses; Troisième édition, á laquelle se trouve joint un épitomé de principes fondamentaux de l'économie politique: Par Jean-Baptiste Say, Chevalier de Saint-Wolodomir, membre de l'Académie des Sciences de Saint-Pétersbourg, de celle de Zurich, etc.; Professeur d'Économie Politique á l'Athénée de Paris, París, De l'Imprimerie de Crapelet, 2 tomos, 1817.

53 Tratado de Economía Política o exposición simple del modo cómo se forman, distribuyen y consumen las riquezas. Por Juan Bautista Say. Refundido por el mismo y aumentado con un epitome que comprende los principios fundamentales de la economía política y una tabla analítica de materias. Traducido al castellano por Don Manuel María Gutiérrez y Don Manuel Antonio Rodríguez, Madrid, Imprenta de Collado, 3 tomos, 1817.

54 Tratado de Economía Política o exposición simple del modo cómo se forman, distribuyen y consumen las riquezas, escrito en francés por Juan Bautista Say, y traducido al castellano por..., [el tomo 1 publicado en 1804 y el tomo 2 en 1805, ambos en Pedro María Caballero, y el tomo 3 en 1807 en Gómez Fuentenebro y Compañía].

55 «En général, Monsieur le Bachelier, méfiez-vous de ces progrès extraordinaires qu'on dit avoir été faits dans les sciences. M Say, dans la dédicace de son dernier ouvrage a l'empereur de Russie, n'assure-t-il pas que si S. M. I. n'eût daigné venir á Paris avec ses innombrables armées, l'Europe serait condamnée á ignorer encore l'état véritable de la science de l'économie publique? Vous voyez á quoi tiennent les découvertes: ces grandes occasions n'arrivent pas tous les jours... C'est dommage! Monsieur, cet esprit-là ne ressemble pas mal á de la satire: Si M Say a dit une pareille chose il a sans doute eu de bonnes raisons; c'est un homme de mérite; ses ouvrages, quoique mal traduits en Espagne, y sont en grande vénération, et je vous assure que cette partie des connaissances humaines n y est pas du tout négligée.» Mercure de France (1 de febrero de 1817), p. 119. Jean Baptiste Esménard era el especialista sobre España en la revista y firmaba los artículos sobre Historia o Política, mientras el detentor del pseudónimo «Le Bachelier de Salamanque» anunciaba sin mucha moderación las noticias no oficiales. 
sanos principios se han difundido, y hecho bastante comunes, aunque queden todavía algunas personas de aquellas que parecen estár condenadas á ignorar siempre la ciencia; y que encaprichadas con su sistema agrícola, con la nulidad del comercio, con su sistema fiscal, y su quimerica balanza del comercio, no quieren escuchar cosa alguna que pueda avergonzarlos de sus rancios errores; pero por fortuna son conocidas y despreciadas.

En cuanto al juicio que hace el Sr, Bachiller del merito de la traduccion, aunque me parece que por la fecha en que se escribió, habla del Sr. Queypo, lo miro como absolutamente falso; porque es una de las traducciones, en mi concepto, que le han hecho en este pais con mas atencion y detenimiento. Así, me créo obligado en su ausencia á defenderle cuando se me presente la ocasión, y á lavar tambien á mi maestro de lo que se le quiere hacer decir y nunca ha dicho.

No haria lo mismo, si se atacase á mi opinion, porque acostumbro á mirar con indiferencia estos juicios que por lo comun son de personas que entienden poco las materias de que juzgan. Yo no aspiro a otra cosa que a merecer la aprobación de V.

A este fin he puesto en manos de los Señores Aguirre [,] del comercio de esta plaza ${ }^{56}$, un ejemplar completo de mi traduccion en 4 volumenes en pasta. Los 3 primeros comprenden su traducción, y el $4^{\circ}$ el epitome y la cartilla, pues no he tenido noticia de la dirección que se ha dado al otro ejemplar que remití á Bayona.

Los Señores Aquirre Hermanos están en correspondencia con los Señores Delaroche Dilassent [sic] y Compañia ${ }^{57}$ pero no es posible ponerle en sus manos, á causa de la poca ó ninguna comunicación que hay con Havre de Grace $^{58}$. Ruego que V. la reciba y la lea, estimaré que me dé su dictamen para aprovecharme de sus observaciones en la $3^{\mathrm{a}}$ edición.

He recibido y leido con mucho gusto el opusculo de que me ha hecho V. espresion, Sobre la Situación Económica de la Inglaterra ${ }^{59}$. Lleva el sello de exâctitud y precisión que distingue cuanto sale de su pluma y siento no poder leer su discurso de obertura pronunciado en ése Atenéo.

Luego que recibí la de V. escribí á la Sociedad Economica de Madrid solicitando, que nombrase á V. miembro de aquel cuerpo, y le daré parte de las resultas.

56 Los hermanos Mariano y Mauricio Aguirre se trasladaron desde Bilbao a Santander en 1797, formando la sociedad Aguirre Hermanos de Santander con un capital de 672.556 reales de vellón. Se trata de un caso más de comerciantes atraídos por la apertura al comercio de ultramar del puerto de Santander y al lucrativo negocio del envío de harinas castellanas. Ver Moreno Lozano (1999).

57 Say tiene una estrecha relación con Michel Delaroche, para quien su hijo Horace trabajaba en Nantes desde 1813, y con la familia Delessert. También era titular de una cuenta bancaría con Delaroche, Delessert et Cia para operaciones de crédito e inversión (Schoorl 2012, pp. 55-59).

58 Le Havre, Normadía.

59 De l'Angleterre et des Anglais, par Jean-Baptiste Say, auteur del Traité d'économie politique, Par Jean-Baptiste Say, Auteur du Traité d'Économie politique (1815). 
En tanto pueda usted mandar con toda dignidad y confianza, á su atento y apasionado servidor.

V.B.S.M

P.D.

Manuel María Gutierrez

Me he tomado la libertad de escribir á V. en mi lengua, porque me cuesta bastante trabajo espresarme en la suya.

El Sr. Consul M. Graslin disginará á V. la persona de confianza á quien podrá entregarle su contestación, y la misma queda encargada de recibir y trasmitirle las ordenes que en adelante me quiéra dar.

\subsection{Carta tercera de Manuel María Gutiérrez}

Señor Dn. Juan B. ${ }^{a}$ Say

Muy Sr. Mío. Acompaño á V. original de la que acabo de recibir de Madrid de un amigo, miembro de la Sociedad Económica, por la cual verá V. que ha quedado admitido con unanimidad de votos [por] ser Socio correspondiente $^{60}$. He tenido en ésto una satisfaccion, tanto por ver reconocido y recompensado su merito, como por haber tenido esta ocasión de manifestar á V. mi estimacion, y los deseos que tengo de complacerle.

Hace yá algun tiempo que está en Bayona, segun se me avisa, un ejemplar completo encuadernado en pasta de la traduccion de su Tratado de Economia politica, Epitome y Catecismo $2^{\mathrm{a}}$ edicion, y créo que no tardará V. mucho tiempo en recibirle.

Continúo limando, corrigiendo, y traduciendo el mismo tratado, con presencia de su $3^{\mathrm{a}}$ edicion, é inmediatamente que se concluya y publique, enviaré á V. por medio del mismo Sr. Consul General de éstas tres provincias, un egemplar completo.

Dios guarde á V. muchos años. Santander 25 de julio de 1817

B.L.M. de Ve. Su mas atento servidor Manuel María Gutierrez

\subsection{Carta de Álvaro Flórez Estrada}

Paris 19 de septiembre de $1831^{61}$

Monsieur J. B. Say, membre du Conseil General de la Seine

${ }^{60}$ José Felipe de Olive.

61 Tras el trienio liberal, Álvaro Flórez Estada vuelve al exilio. Su primer destino será Londres aunque desde octubre de 1830 hasta 1834 reside en París. El 9 de mayo de 1834 vuelve a España (Uría Ríu 1955, pp. 39-76). 
Mui Señor mio de todo mi estimacion y respeto: la aprobacion que V. hace de mi Obra de Economia ${ }^{62}$, en su apreciable carta de fecha de antes de ayer, aunque concebida en terminos mas favorables de lo que merece, es el testimonio mas lisongero que podria recibir acerca del resultado de mis trabajos, no teniendo que desear en esta parte, una vez obtenido el voto de una persona de la reputacion, y conociminetos de V. en la materia.

Agradezco la atencion que V. me indica de remitirme a haberle tenido un exemplar de la ultima edicion de su Obra pero debiendo yo dar á luz nimi [i] luego una tercera de la mia bastante aumentada, necesitaré hacerme con ella, asi para aprovecharme de la luces que espero hallar en los nuevos desarrollos, que V. le ha dado, como para hacer mencion de ellos ${ }^{63}$.

Creo seguramente de la mayor importancia impugnar, como V. se propone hacerlo en el mes del proximo Noviembre, la preocupacion de que refluyen á la sociedad los consumos que hacen los Gobiernos, pues aunque no es la idea menos demostrada, desgraciadamente es la mas resistida por lo interesados en los abusos. Como espero pasar el invierno en Paris, no perderé la oportunidad de asistir á sus lecciones, de que espero reportar no poco provecho y satisfaccion ${ }^{64}$.

Deseo que V. logre ver el triunfo de su sabia Doctrina, y que me conserve en su amistad y memoria, asegurandole que mi voluntad y persona estarán siempre prontas á complacerle, en cuanto ordenare á su afectísimo y atento servidor.

Alvaro Florez Estrada

\subsection{Carta del Marqués de Valle Santoro}

Sr. J.B. Say:

Señor: entregado desde hace algunos años al estudio de la economía política, soy uno de los justos admiradores de su obra; pero como es fácil que una misma cuestión sea considerada por dos personas de una manera distinta, me tomo la libertad de indicaros cuales son mis ideas sobre la libertad absoluta del Comercio, que es un objeto de gran importancia para el interés de todos los pueblos ${ }^{65}$.

${ }^{62}$ Curso de Economía política, Segunda edición corregida y aumentada considerablemente. París, Imp. De Gaultier-Laguionie, 2 vols., 1831.

${ }^{63}$ La tercera edición del Curso es una traducción al francés de Leon Galibert, con el título Cours eclectique d'economie politique (París, Treuttel et Würtz, 3 vols., 1833). Flórez había revisado de forma más importante esta tercera edición con cambios en la teoría de la renta y en la dinámica económica a largo plazo (Almenar 2000, pp. 369-411).

${ }^{64}$ En 1830, Say es nombrado primer titular de la Cátedra de Economía política del Collège de France. Imparte únicamente el primer curso académico completo porque fallece en noviembre de 1832. En la primera edición de sus obras completas de 1848 aparecen publicados las dos lecciones inaugurales que inauguraban los años académicos 1831-22 y 1832-33.

65 Será una cuestión a la que dedica una obra en particular. Tras la notoriedad alcanzada con las dos ediciones de sus Elementos de Economía política aplicados a España, Francisco de Gregorio, Marqués del Valle Santoro, publica en 1830 Memoria sobre la balanza de comercio y estado de la 
Usted Señor se decantó por la libertad absoluta del comercio y se fundó principalmente en que hay siempre intercambio de igual valor, pues sin esto no habría comercio. En consecuencia, usted deduce que cuando dos naciones comercian conjuntamente ambas ganan a la vez, que era un error creer que una ganaba a costa de la otra y que, por lo tanto, el sistema restrictivo es no solo inútil, sino muy nocivo ${ }^{66}$.

Al principio, cuando se intercambian objetos de igual valor es tan evidentemente verdad que nadie ha podido refutarlo. Pero la experiencia de tres siglos ha probado que las naciones que han seguido el sistema restrictivo se han enriquecido mucho más rápido y han adquirido un grado de poder mucho mayor que aquellas que lo han obviado. Por esto me he aplicado a encontrar las causas que han podido contribuir a ello y a combinar este hecho con su teoría para obtener las consecuencias que pudieran servir para solucionar la cuestión.

Creo Señor que la ventaja de las naciones que han adoptado el sistema restrictivo ha dependido en gran parte de lo que había en otras y que a ellas le faltaban; ventaja que ha cesado en el momento que todas lo han adoptado y cuyo resultado habría sido la destrucción de todo comercio.

El sistema restrictivo tenía como base no permitir otra entrada que las materias primas y fomentar la venta de productos de la industria. Es necesario observar que los primeros son productos agrícolas en los que la naturaleza tiene mucha importancia para la producción, mientras los otros son principalmente debidos al trabajo de los hombres. Así, para obtener los primeros son necesarios muchos terrenos y menos brazos que para obtener los segundos. En consecuencia, las naciones que dan productos agrícolas a cambio de productos industriales deberían quedar muy atrás en relación con aquellos otros, ya sea en población o en agricultura ${ }^{67}$.

Polonia, por ejemplo, dando trigo, debido en parte a la naturaleza, y recibiendo objetos manufacturados no puede estar tan poblada como aquellos que le dan estos objetos, ni tampoco tener tan brillante agricultura porque el aumento de los hombres que requiere la industria le exige otra agricultura para mantenerlos; y es un hecho que en ningún sitio la agricultura es tan brillante como en los alrededores de una ciudad manufacturera; en comparación con un terreno fértil sin ciudades industriosas.

En consecuencia, la nación que da objetos de industria debería crecer mucho más rápido en todo tipo de producciones que aquellas que dan

\footnotetext{
(footnote continued)

riqueza actual en España. Serrano (2012) considera que se trata de uno de los primeros libros que trataron el sector exterior de la economía española.

66 En la Memoria sobre la balanza de comercio (1830), Valle Santoro reproduce varios extractores del capítulo XVII del libro primero de Tratado de Economía Política. Emplea la traducción de la cuarta edición publicada en 1821 en Madrid por Juan Sánchez Rivera (Serrano 2012, p. 38).

67 Valle Santoro (1830, p. 199).
} 
productos agrícolas, pues cuando se crece en población y en agricultura se crece también en riqueza, según sus principios mismos.

Para presentaros un ejemplo más sorprendente aún examinaremos el comercio que España ha hecho durante estos mismos siglos con Inglaterra; la primera da lana y la segunda objetos de industria. Hay siempre intercambio de igual valor, pues sin estos no habría comercio; pero para obtener este valor en lana uno abandonó y dejó sin cultivo dos provincias inmensas, empleando para obtenerlo únicamente un pequeño número de pastores que a su vez tenían necesidad de poca agricultura para su subsistencia, mientras Inglaterra obtiene sus productos de un pequeño espacio de terreno empleando a un gran número de personas, que a su vez exigen mucha agricultura para su sostenimiento. Por lo tanto, resulta una progresión siempre creciente, sea en población o sea en agricultura, y en consecuencia en la riqueza pública ${ }^{68}$. Por esto, juzgo Señor cuanto era más ventajoso este comercio para Inglaterra que para España y cuanto queda más retrasado este que el otro.

En consecuencia, concluyo que el comercio interior en toda nación debe ser libre y que el comercio general debería serlo también, si todas las naciones tuvieran un único interés. Pero como no es siempre el mismo, creo que la base de la tarifa de las aduanas debe ser el sistema restrictivo, salvo que cada nación haga tratados de comercio en los que se aproximen todo lo posible a la libertad absoluta de comercio mediante concesiones mutuas y en función de las circunstancias particulares de cada una de ellas ${ }^{69}$.

Reciba Señor les sentimientos de la más alta consideración con la que tengo el honor de ser su muy humilde y muy dispuesto servidor.

El Marqués de Valle Santoro

\section{REFLEXIONES FINALES}

Esperamos que este trabajo contribuya a desterrar ciertas visiones que, partiendo de la negación de la existencia de un avance intelectual significativo en la España del setecientos y la primera mitad del ochocientos, venían a atribuir la abundante presencia de traducciones a la debilidad de la literatura propia, a la dependencia respecto a otros países o a simples modas reprobables (Lafarga 1999, 12-14). La correspondencia publicada demuestra que las abundantes traducciones en España, al contrario de actuar en detrimento de las propias publicaciones españolas, acercaron a los autores españoles al mundo académico de referencia.

Más allá de encontrar las respuestas de Say a estas cartas españolas, queda por desarrollar y concretar el proyecto colectivo de traducción de la

\footnotetext{
68 Valle Santoro (1830, p. 199).

69 Valle Santoro (1830, p. 198).
} 
obra Say, fraguado en la Real Sociedad Económica Matritense de Amigos del País y hacia el que apunta la correspondencia de Gutiérrez. Es posible que este camino nos permita conocer por qué el Traité será la obra recomendada como libro de texto desde 1807 y qué relación hay entre esta elección y los debates políticos sobre la reforma universitaria que le fueron contemporáneos. También será necesario indagar en las estructuras que facilitan la circulación de las ideas, y no solo en aquellas que la dificultaron. Esta correspondencia señala tanto el papel de la información periodística en el inicio de la difusión como los canales diplomáticos y financieros para la circulación de ejemplares.

\section{BIBLIOGRAFÍA}

Albertone, M. y Masoero, A. (1994): Political Economy and National Realities. Torino: Fondazione Luigi Einaudi.

Almenar, S. (1996): "Economic Thought in Spanish Periodical Publications of the 18th and the 19th Centuries: An Introduction», History of Economic Ideas 4 (3), pp. 119-147.

Almenar, S. (2000): «Álvaro Flórez Estrada y la economía política clásica», en E. Fuentes Quintana (coords.) Economía y economistas españoles, vol. 4. Madrid: Galaxia Gutenberg, pp. 369-411.

Antón Ramírez, B. (1865): Diccionario de bibliografía agronómica. Madrid: Imprenta de M. Rivadeneyra.

Astigarraga, J. (2000): «Necker en España, 1780-1800», Revista de Economía Aplicada 8 (23), pp. 119-141.

Astigarraga, J. (2004): «Diálogo económico en la 'otra' Europa. Las traducciones españolas de los economistas de la Ilustración napolitana (A. Genovesi, F. Galiani y G. Filangieri)», Cromohs 9, pp. 1-21.

Astigarraga, J. y Zabalza, J. (2007): «La fortuna del Essai sur la nature du commerce en général (1755), de Richard Cantillon, en la España del siglo XVIII», Investigaciones de Historia Económica 3 (7), pp. 9-36.

BLADÉ, J.-F. (1870): «Examen critique d'un livre intitulé étude sur l'origine des basques», Revue critique d'histoire et de littérature 12-13, pp. 19-26.

Bolufer, M. (2003): «Civilización, costumbres y política en la literatura de viajes a España en el siglo XVIII», Estudis 29, pp. 255-300.

CARDOSO, J. L. (2003): "The international diffusion of economic thought», en W. J. Samuels; J. E. Biddle y J. B. Davis (eds.) A Companion to the History of Economic Thought. Malden MA: Blackwell Publishing, pp. 622-633.

Carpeter, K. E. y Redlich, F. (1973): «Research Possibilities in the History of Political Economy Thought: a Bibliography of Translations», History of Political Economy 5 (1), pp. 268-283.

Castro Valdivia, M. (2013): «La obra de Jean-Baptiste Say: Traducciones e influencias en la primera mitad del siglo XIX». Lisboa: VIII Encuentro ibérico de historia del pensamiento económico.

Colander, D. C. y Coats, A. W. (1989): The Spread of Economic Ideas. Nueva York: Cambridge University Press.

Defourneaux, M. (1973): Inquisición y censura de libros en la España del siglo XVIII. Madrid: Taurus. 
Domergue, L. (2003): «El escritor y el Estado en tiempos de las Luces», en A. Morales Moya (ed.) 1802. España entre dos siglos. Sociedad y Cultura. Madrid: Sociedad Estatal de Conmemoraciones Culturales, pp. 263-272.

Hall, P. A. (1989): The Political Power of Economic Ideas: Keynesianism across Nations. Princeton: Princeton University Press.

Hirschman, A. O. (1989): «How the Keynesian Revolution Was Exported from the United and Other Comments», en P. A. Hall (ed.) The Political Power of Economic Ideas: Keynesianism across Nations. Princeton: Princeton University Press, pp. 347-359.

Hoyos, J. C. (2015): «Nacimiento de la ciencia económica: análisis de las traducciones españolas del Epítome de Jean-Baptiste Say». En J. Pinilla y B. Lépinette (eds.) Traducción y difusión de la ciencia y de técnica en España (s. XVI-XIX). Valencia: Publications de la Universitat de València, pp. 295-314.

Iglesias, C. (1998): "España desde fuera», en E. Benito (coord.), España. Reflexiones sobre el ser de España. Madrid: Real Academia de la Historia, pp. 377-428.

LAFARGA, F. (1999): «Historia de la traducción en España (1750-1830)», en F. Lafarga (ed.) La traducción en España (1750-1830). Lengua, Literatura, Cultura. Lleida: Universitat de Lleida, pp. 11-31.

Lasarte, J. (1976): "Adam Smith ante la Inquisición y la Academia de la Historia», en E. Fuentes Quintana (coords.) Economía y economistas españoles, vol. 4. Madrid: Galaxia Gutenberg, 2000, pp. 239-268.

Llombart, V. (2004): «Traducciones españolas de economía política (1700-1812): catálogo bibliográfico y una nueva perspectiva», Cromohs 9, pp. 1-14.

Lluch, E. (1980): "Sobre la historia nacional del pensamiento económico», en S. Almenar (ed.) Curso de economía política por D. Álvaro Flórez Estada. Madrid: Instituto de estudios fiscales, pp. 15-62.

López, F. (1995): «El libro y su mundo», en J. Álvarez Barrientos (ed.) La república de las letras en la España del siglo XVIII. Madrid: CSIC, pp. 63-123.

López Castellano, F. (2009): «La réception de Say et son influence sur l'institutionnalisation de l'enseignement de l'économie en Espagne (1807-1856)», Revue d'Histoire des Sciences Humaines 21, pp. 127-150.

Martín Rodríguez, M. (1989): «La institucionalización de la Economía política en la Universidad Española (1784-1857)», en F. G. Marqués del Valle Santoro Elementos de Economía política con aplicación particular a España. Madrid: Instituto de Estudios Fiscales, pp. IX-CCXXXVII.

Menudo, J. M. y O'Kean, J. Ma . (2005): «La recepción de la obra de Jean-Baptiste Say en España: la teoría económica del empresario», Revista de Historia Económica Journal of Iberian and Latin American Economic History 23 (1), pp. 117-142.

Moral, A. M. y Colmenero, R. (2007): «Un espacio reformista en el Madrid de FernandoVII: la Real Sociedad Económica Matritense de Amigos del País (1814-1833)», Madrid. Revista de arte, geografía e historia 8, pp. 87-127.

Moreno Lozano, J. (1999): Los empresarios harineros castellanos (1765-1913). Madrid: Fundación Empresa Pública.

Montagut, E. (2004): «Las cartillas agrarias en la crisis del Antiguo Régimen», Torre de los Lujanes 52, pp. 87-101.

Ramos Gorostiza, J. L. (2006): «La imagen económica de la España de Carlos III: Joseph Townsend, Alexander Jardine y los economistas españoles», Revista de Historia Económica - Journal of Iberian and Latin American Economic History 24 (1), pp. 139-173.

ReEder, J. (1978): «Economía e Ilustración en España: Traducciones y traductores 1717-1800», Moneda y Crédito 147, pp. 47-70. 
SAncha, A. (1780): Memorias de la Sociedad Económica, Vol. 2. Madrid: Real Sociedad Económica Matritense de Amigos del País.

SAY, J. B. (1814): Traité d'économie politique, ou Simple exposition de la manière dont se forment, se distribuent et se consomment les richesses; Seconde édition entièrement refondue et augmentée d'un épitomé des principes fondamentaux de l'économie politique: Par Jean-Baptiste Say, ex-membre du Tribunat. París: De l'Imprimerie de Crapelet.

SAY, J. B. (1816): Tratado de economía política ó exposición sencilla de como se forman, se distribuyen y se consumen las riquezas. Traducción e introducción de Manuel María Gutiérrez y Manuel Antonio Rodríguez. Madrid: Imprenta de Collado.

SAY, J. B. (1821): Tratado de economía política ó exposición de cómo se forman, se distribuyen y se consumen las riquezas. Madrid: Fermín Villalpando.

SAY, J. B. (1840): Cours complet d'économie politique pratique et mélange et correspondance d'économie politique. Roma: Edizione dell'Ateneo Bizzarri, 1968.

SAY, J. B. (2006): Jean-Baptiste Say. Euvres complètes I: Traité d'économie politique ou simple exposition de la manière dont se forment, se distribuent et se consomment les richesses. Édition variorum des six éditions. París: Economica.

ScHoorl, E. (2012): Jean-Baptiste Say: Revolutionary, Entrepreneur, Economist. Londres: Routledge.

Schwartz, P. y Rodríguez Braun, C. (1983): "Cartas españolas de Jeremías Bentham», Moneda y Crédito 165, pp. 59-88.

Serrano, J. M ${ }^{\mathrm{a}}$. (2012): "Francisco de Gregorio, Marqués del Valle Santoro», en F. G. Marqués del Valle Santoro Hacienda Pública-Balanza del comercio. Zaragoza: Institución Fernando el Católico, pp. 11-54.

SPENGLER, J. J. (1970): «Notes on the International Transmissions of Economics Ideas», History of Political Economy 4 (2), pp. 133-151.

Steiner, Ph. (1990): «L'économie politique pratique contre les systèmes: quelques remarques sur la méthode de J.-B. Say», Revue d'économie politique 100 (5), pp. 664-678.

STEINER, Ph. (1998): «The structure of Say's economic writings», The European Journal of the History of Economic Thought 5 (2), pp. 227-249.

Uría Ríu, J. (1955): «Flórez Estrada en París, 1830-1834», Archivum. Revista de la Facultad de Filosofía y Letras 5 (1), pp. 39-76.

Valle SAntoro, F. G. M. de (1830): Memoria sobre la balanza de comercio y estado de la riqueza actual en España, en F. G. Marqués del Valle Santoro Hacienda PúblicaBalanza del comercio. Zaragoza: Institución Fernando el Católico, 2012. 